\title{
Effect of EcoR1 Polymorphism of the Apoprotein B100 Gene (Apo B 4154G>A) on Serum Lipid Profile in ART-Naive PLHIV
}

\author{
Kone-Kone Fatoumata ${ }^{1,2, *}$, Aka Tano Cyrielle ${ }^{2}$, Kone-Dakouri Yekayo ${ }^{1}$, Toni Thomas D. Aquin ${ }^{1,3}$, \\ Konan Konan Jean Louis ${ }^{1}$, Ahiboh Hugues ${ }^{1,2}$, Yayo Sagou Eric ${ }^{1}$, Edjeme Ake Angele ${ }^{1}$, \\ Attoungbre-Hauhouot Marie Laure ${ }^{1}$, Djaman Joseph ${ }^{4}$, Monnet Dagui ${ }^{1}$ \\ ${ }^{1}$ Department of Biochemistry, Molecular Biology, Reproduction Biology and Medical Pathologies, Unit of Education and Research of \\ Pharmaceutical and Biological Sciences, Felix Houphouet Boigny University, Abidjan, Ivory Coast \\ ${ }^{2}$ Diagnostic and Research Center on AIDS and Other Infectious Diseases (CeDReS), Treichville University Hospital, Abidjan, Ivory Coast \\ ${ }^{3}$ Integrated Bioclinical Research Center (CIRBA) of Abidjan, Abidjan, Ivory Coast \\ ${ }^{4}$ Department of Biochemistry, Unit of Education and Research of Biosciences, Felix Houphouet Boigny University, Abidjan, Ivory Coast
}

\section{Email address:}

kaunefatou@yahoo.fr (Kone-Kone F.),cyrielleaka@gmail.com (Aka T. C.), bene.yekayo@yahoo.fr (Kone-Dakouri Y.), ta_toni@yahoo.com (Toni T. D. A.), hugahibo@gmail.com (Ahiboh H.), angelake@aviso.ci (Edjeme Ake A.), yayodid@yahoo.fr (Yayo S. E.), mlattoungbre@hotmail.com (Attoungbre-Hauhouot M. L.), djamanj@yahoo.fr (Djaman J.), monnetdagui@hotmail.com (Monnet D.)

${ }^{*}$ Corresponding author

\section{To cite this article:}

Kone-Kone Fatoumata, Aka Tano Cyrielle, Kone-Dakouri Yekayo, Toni Thomas d'Aquin, Konan Konan Jean Louis, Ahiboh Hugues, Yayo Sagou Eric, Edjeme Ake Angele, Attoungbre-Hauhouot Marie Laure, Djaman Joseph, Monnet Dagui. Effect of EcoR1 Polymorphism of the Apoprotein B100 Gene (Apo B 4154G>A) on Serum Lipid Profile in ART-Naive PLHIV. Biochemistry and Molecular Biology.

Vol. 6, No. 3, 2021, pp. 48-57. doi: 10.11648/j.bmb.20210603.13

Received: June 28, 2021; Accepted: July 12, 2021; Published: July 23, 2021

\begin{abstract}
Many people living with HIV (PLHIV) have dyslipidemia and influence of genetic predisposing factors are suspected. Some apolipoprotein genes polymorphisms are recognized as susceptibility factors, especially EcoR1 polymorphism of Apo B100 gene, main atherogenic lipid metabolizing apoprotein. The objective was to investigate the link between EcoR1 polymorphism of Apo B100 gene (Apo B 4154G>A) and the occurrence of dyslipidemia in ART-naive PLHIV. We did a cross-sectional study which included $32 \mathrm{HIV-negative} \mathrm{controls} \mathrm{and} 23$ PLHIV, above 18 years old, with at least one serum lipid and apoprotein disorder. Polymorphism testing was performed by PCR-RFLP and allele distribution study was performed using Hardy Weinberg equilibrium. As results, we found that the subjects were predominantly young males in both groups. BMI was higher in PLHIV. There were lipid disorders common to both PLHIV and HIV- subjects. However, PLHIV were distinguished by hypoapoproteinemia Apo A1 and HDL hypocholesterolemia. The 3 possible genotypes of EcoR1 polymorphism were found in both groups with a predominance of the mutant genotype in PLHIV (85.7\% vs $14.3 \%)$. Also, the mutant allele frequency was higher in PLHIV (27.1\% vs 6.2\%). Regardless of HIV status, the mutant allele was more frequent in people under 40 years old, women and people with high BMI. In PLHIV, Hardy Weinberg equilibrium was deviated in all subgroups with the mutant allele frequency higher than $10 \%$. In the overall population, the mutant allele was more frequent in subjects with increased TG, LDL-C, Apo B100, Lp (a), and CT/HDL atherogenicity index and those with normal total cholesterol, decreased HDL-C and Apo A1. Taking into account HIV status, the mutant allele found was more frequent in PLHIV (14\% to $32 \%$ versus $2 \%$ to $21 \%$ ). In these PLHIV, the mutant allele was more frequent especially in cases of total hypercholesterolemia (28.1\%), normal LDL cholesterolemia (26.7\%), HDL hypocholesterolemia (27.3\%), hypoapoproteinemia A1 (32.1\%), hyperapoproteinemia B100 (28.1\%), hyperapoproteinemia Lp (a) (28.1\%), high atherogenicity indexes (23.7\%). In conclusion, the distribution of EcoR1 polymorphism alleles at position 4154 in exon 29 of ApoB100 gene was not random in PLHIV people. The carrying of mutant allele was more frequent in PLHIV and associated to increased atherogenic apolipoproteins and decreased atherosclerotic protective apolipoproteins (Apo A1 and HDL-C). The link between EcoR1 and ART must be investigates.
\end{abstract}


Keywords: EcoR1 Polymorphism, Apolipoprotein B, Dyslipidemia, HIV, Restriction Enzymes

\section{Introduction}

Human immunodeficiency virus (HIV) infection remains a public health problem. In 2020, 37.6 million [30.2- 45.0 million] people were living with HIV worldwide [1]. Infected persons, treated or not by antiretroviral drugs, show metabolic disorders, dyslipidemia in particular, a risk factor for cardiovascular diseases [2, 3, 4]. This suggests that the infection itself has a deleterious metabolic effect $[3,5,6]$. Also, all PLHIV do not have dyslipidemia, raising the suspicion that host-related genetic predisposing factors have an influence on the occurrence of dyslipidemia. Several single nucleotide polymorphisms (SNPs) were associated with the occurrence of dyslipidemia, particularly in the apolipoprotein genes $[7,8$, 9, 10]. It has been shown that HIV-associated dyslipidemia is accompanied by an increase in lipoproteins containing Apolipoprotein B (Apo B), particularly LDL, and high LDL cholesterol is known to be one of the predictive factors of cardiovascular risk [3,5]. Thus, the Apo B100 gene is one of the most studied genes of interest; particularly the EcoR1 and Xbal polymorphisms $[7,10,11]$.

The objective of this study was to establish the link between EcoR1 polymorphism of the Apo B gene and changes in serum lipoprotein profiles in ART-naive PLHIV in Côte d'Ivoire.

\section{Materials and Methods}

\subsection{Materials, Population and (Setting) Study Sites}

The study population consisted of HIV-negative controls (HIV-) voluntary blood donors and PLHIV monitored at the National Blood Transfusion Center (CNTS) management center and showing a disturbed lipid report (classical lipids and apolipoproteinemia).

\subsection{Methods}

This was a cross-sectional study.

Sample collection and storage

Whole blood collected on EDTA tubes was sent to CeDReS where aliquots were stored at $-80^{\circ} \mathrm{C}$, and then lipoprotein parameters were measured. Sociodemographic, therapeutic and biological data (lipid report) were collected from the database of the first part of the study. Indeed, the first part of the study had already been carried out using these same samples [12, 13].

Ethical considerations

The recruited persons gave their informed consent to participate in the study. The study was conducted with the approval of the National Ethics and Research Committee of Côte d'Ivoire under the reference number: 3766/MSHP/30/July 2009.

Determination of the EcoR1 polymorphism of the ApoB100 gene

Primers used: The primer pair (SIGMA ALDRICH) used for PCR was: $\mathrm{Bc} 2 / \mathrm{Bc} 2 \mathrm{R}$ (sequence of interest on exon 29).
The sequences were: Bc2: lot HA06611951 (5'-GAAGAG CCTGAAGACTGACT-3'), Bc2R: lot HA06611952 (5'CTCGAAAGGAAGTGTAATCAC-3').

Extraction and study of extracts: The extraction of genomic DNA was performed on globular pellets with the Qiagen ${ }^{\circledR}$ kit technique. Extracts were stored at $4{ }^{\circ} \mathrm{C}$ until amplification and then the rest frozen at $-80^{\circ} \mathrm{C}$ for later use.

Amplifications: Amplifications were done by conventional PCR and the size of the sequence of interest was $480 \mathrm{bp}$. The PCR was performed with a $50 \mu \mathrm{L}$ reaction mixture consisting of $1.5 \mu \mathrm{L}$ of each of the primers at $10 \mu \mathrm{M}, 5 \mu \mathrm{L}$ of solution containing the $4 \mathrm{dNTPs}$ at $2 \mu \mathrm{M}, 1.5 \mu \mathrm{L}$ of $\mathrm{MgCl} 2$ at $50 \mu \mathrm{M}, 5$ $\mu \mathrm{L}$ of $10 \mathrm{X}$ buffer, $0.5 \mu \mathrm{L}$ of Taq polymerase at $5 \mathrm{IU} / \mu \mathrm{L}, 30 \mu \mathrm{L}$ of molecular biology grade water and $5 \mu \mathrm{L}$ of DNA PCR was performed on an ABI 2720 Thermal Cycler (Life Technologie). The PCR program consisted of an initial denaturation for $5 \mathrm{~min}$ at $95^{\circ} \mathrm{C}$ followed by 40 cycles of the following 3 steps: denaturation for $45 \mathrm{sec}$ at $95^{\circ} \mathrm{C}$, hybridization at $58^{\circ} \mathrm{C}$ for $45 \mathrm{sec}$ and elongation at $72^{\circ} \mathrm{C}$ for $45 \mathrm{sec}$ followed by a final elongation at $72{ }^{\circ} \mathrm{C}$ for $10 \mathrm{~min}$ and finally $4^{\circ} \mathrm{C}$ ad infinitum $[12,14]$. Success of PCR was verified by electrophoresis on $1 \%$ agarose gel containing ethidium bromide (BET). In case of failure (no band on electrophoresis), the PCR was repeated. The amplicons were stored in the refrigerator $\left(4-8^{\circ} \mathrm{C}\right)$ pending the RFLP step.

Enzymatic digestion and the search for polymorphism: After PCR, the amplification products were subjected to digestion with the restriction enzyme EcoR1 (G / AATTC) according to the predefined protocol [10]. After the digestion step, the DNA fragments were separated by electrophoresis on $1 \%$ agarose gel containing ethidium bromide (BET). The action of the enzyme generates 2 fragments of size 253 and $227 \mathrm{bp}[11,15]$ and indicates the presence of the $\mathrm{G}$ (wild type) allele of the $4154 \mathrm{G}>\mathrm{A}$ polymorphism of exon 29 of the ApoB100 gene (E+ allele). The absence of digestion of the sequence indicates the A allele (mutant) and results in a single $480 \mathrm{bp}$ fragment $\mathrm{G}$ allele (wild).

Study of the distribution of alleles: It has been made by the Hardy-Weinberg equilibrium (HWE). Allelic frequencies are determined from the observed genotypic frequencies. Theoretical numbers of each genotype are then calculated and compared with the observed numbers from the chi-square $\left(\mathrm{X}^{2}\right)$ calculation. The $\mathrm{X}^{2}$ is significant if $\mathrm{X}^{2}>3.84[16,17,18]$. Formulas used are:

Allelic frequencies:

$$
\begin{aligned}
& \mathrm{p}(\mathrm{E}+)=(2 \mathrm{x}+\mathrm{z}) / 2 \mathrm{~N} \\
& \mathrm{q}(\mathrm{E}-)=(2 \mathrm{y}+\mathrm{z}) / 2 \mathrm{~N}
\end{aligned}
$$

Expected numbers:

$$
\mathrm{E}+/+=\mathrm{Np}^{2} ; \mathrm{E}-/-=\mathrm{Nq}^{2} ; \mathrm{E}+/-=2 \mathrm{pqN}
$$


Calculated Chi-square:

$X^{2}=\sum\left[(\text { observed numbers - expected numbers })^{2} /\right.$ expected numbers $]$

$\mathrm{N}$ : size of the study population, $\mathrm{x}$ : number of individuals with $\mathrm{E}+/+$ genotype, y: number of individuals with $\mathrm{E}-/-$ genotype,

z: number of individuals with $\mathrm{E}+/$ - genotype

Statistical analysis of data

The collected data were analyzed using SPSS 16.0 software. The presence of DNA digestion was noted $(+)$ and its absence (-). Quantitative variables were expressed on average \pm standard deviation. Qualitative variables were expressed as percentages (\%) and numbers (n). Comparisons were made using statistical tests: Student t-test for quantitative values and Chi 2 or Fisher exact test for qualitative values. The tests were considered significant at the risk $\alpha<0.05$.

Hardy-Weinberg equilibrium (HWE) was tested by the 1-degree-of-freedom (1ddl) Chi-square test; the significance level was $3.84\left(\mathrm{X}^{2}>3.84\right)$.

\section{Results}

A total of 55 subjects were recruited: 23 ART-naive PLHIV (41.82\%) and 32 HIV- control subjects (58.18\%).

\subsection{Presentation of the Population According to Sociodemographic Cardiovascular Risk Factors}

There was a male predominance in both groups (sex ratio $\mathrm{M} / \mathrm{F}$ : 4.3 in HIV- subjects and 1.3 in PLHIV) but there were no significant differences between the 2 groups regarding gender and age distribution. The mean BMI was higher in PLHIV than in HIV- subjects: $23.82 \pm 3.37$ versus $26.29 \pm 5.4$ $(p=0.046)$. Also, high BMI values $(>25)$ were more frequent in PLHIV: $\mathrm{n}=14(63.6 \%)$ versus $\mathrm{n}=11(35.5 \%)(p=0.043)$.

\subsection{Presentation of the Population According to Serum Lipid and Apoprotein Concentration Levels}

Hypo-apoproteinemia A1 was more frequent in PLHIV $(\mathrm{n}=14(60.90 \%))$ compared to HIV- subjects $(\mathrm{n}=7$ (21.9\%)); $p=0.002$. There was in both groups the predominance of hypertriglyceridemia (PLHIV: 19 (59.4\%); HIV- subjects: $\mathrm{n}=16$ (69.6\%)), hyper-Apo B (PLHIV: 28 (87.5\%); HIVsubjects: $16(69.6 \%)$ ), hyper-apoproteinemia Lp (a) (PLHIV: $\mathrm{n}=20(62.5 \%)$; HIV- subjects: $16(69.6 \%))$ as well as high atherogenicity index: TC/HDL-C (PLHIV: $\mathrm{n}=17$ (53.1\%); HIV- subjects: $n=19(82.6 \%)$ ) and ApoB/Apo A1: (PLHIV: $\mathrm{n}=19 \quad(82.6 \%$; HIV- subjects: $\mathrm{n}=25 \quad(78.1 \%))$. But the difference was significant only for the atherogenicity index TC/HDL-C $(p=0.023)$.

\subsection{Presentation of the Population According to the EcoR1 Polymorphism}

The wild genotype $(\mathrm{E}+/+)$ predominated in both groups and all 3 genotypes were found in both (Table 1). The wild allele $(\mathrm{E}+)$ predominated in HIV- subjects $(p=0.002)$ while the mutant allele (E-) predominated in HIV- subjects (Table
1). The HWE was deviated in both HIV- $\left(\mathrm{X}^{2}=6.97 ; p=\right.$ $0.008)$ and PLHIV subjects $\left(\mathrm{X}^{2}=19.20 ; p<0.0001\right)$.

Table 1. Subject distribution according to geneotypes and alleles.

\begin{tabular}{|c|c|c|c|}
\hline & $\begin{array}{l}\text { HIV-Subject } \\
\mathrm{N}=32\end{array}$ & $\begin{array}{l}\text { ART naive } \\
\text { PLHIV } N=23\end{array}$ & p-value \\
\hline \multicolumn{4}{|l|}{ Genotypes } \\
\hline Wild $(E+/+)$ & $29(63.0 \%)$ & $17(37.0 \%)$ & \multirow{3}{*}{ NA } \\
\hline Mutant / Wild (E+/-) & $2(66.7 \%)$ & $1(33.3 \%)$ & \\
\hline Mutant (E-/-) & $1(16.7 \%)$ & $5(83.3 \%)$ & \\
\hline \multicolumn{4}{|l|}{ Alleles } \\
\hline Wild allele $(\mathrm{E}+)$ & $60(93.8 \%)$ & $35(76.1 \%)$ & \multirow{2}{*}{0.002} \\
\hline Mutant allele (E-) & $4(6,2 \%)$ & $11(23.9 \%)$ & \\
\hline
\end{tabular}

\subsection{Distribution of Genotypes and Alleles According to Cardiovascular Risk Factors}

Regardless of HIV status, genotype frequencies were not at HWE for any of the demographic factors. The mutant allele was more frequent in those under 40 years of age, women, and those with high BMI (Table 2). According to HIV status (Table 3), in the HIV-group, HWE was deviated in those under 40 years of age, males and those with high BMI. In PLHIV, the equilibrium was deviated in all subgroups with frequencies of the mutant allele in the proportions higher than $10 \%$.

Table 2. Genotypes and alleles distribution according to demographic cardio-vascular risk factors in the general population regardless of HIV infection status.

\begin{tabular}{|c|c|c|}
\hline \multicolumn{3}{|l|}{ Age Group $(\mathrm{N}=54)$} \\
\hline & $\leq 40$ years $(n=29)$ & $>40$ years $(n=25)$ \\
\hline \multicolumn{3}{|l|}{ Genotypes } \\
\hline Wild $(\mathrm{E}+/+)$ & $24(53.3 \%)$ & $21(46.7 \%)$ \\
\hline Mutant / Wild (E+/-) & $1(33.3 \%)$ & $2(66.7 \%)$ \\
\hline Mutant (E-/-) & $4(66.7 \%)$ & $2(33.3 \%)$ \\
\hline \multicolumn{3}{|l|}{ Alleles } \\
\hline Allele E+ & $49(84.5 \%)$ & $44(88.0 \%)$ \\
\hline Allele E- & $9(15.5 \%)$ & $6(12.0 \%)$ \\
\hline HWE $\left(X^{2} / p\right.$-value $)$ & $21.87 /<0.0001$ & $9.65 / 0.0019$ \\
\hline \multicolumn{3}{|l|}{$\operatorname{SEX}(\mathrm{N}=55)$} \\
\hline & Male $(n=39)$ & Female $(n=16)$ \\
\hline \multicolumn{3}{|l|}{ Genotypes } \\
\hline Wild $(\mathrm{E}+/+)$ & $33(71.7 \%)$ & $13(28.3 \%)$ \\
\hline Mutant / Wild (E+/-) & $2(66.7 \%)$ & $1(33.3 \%)$ \\
\hline Mutant (E-/-) & $4(66.7 \%)$ & $2(33.3 \%)$ \\
\hline \multicolumn{3}{|l|}{ Alleles } \\
\hline Allele E+ & $68(87.2 \%)$ & $29(85.3 \%)$ \\
\hline Allele E- & $10(12.8 \%)$ & $5(14.7 \%)$ \\
\hline HWE (X²/p-value) & $23.16 /<0.0001$ & $9.31 / 0.002$ \\
\hline \multicolumn{3}{|l|}{$\operatorname{BMI}(\mathrm{N}=53)$} \\
\hline & $\leq 25 \mathrm{~kg} / \mathrm{m}^{2}(\mathrm{n}=28)$ & $>25 \mathrm{~kg} / \mathrm{m}^{2}(\mathrm{n}=25)$ \\
\hline \multicolumn{3}{|l|}{ Genotypes } \\
\hline Wild $(\mathrm{E}+/+)$ & $26(57.8 \%)$ & $19(42.2 \%)$ \\
\hline Mutant / Wild (E+/-) & $1(33.3 \%)$ & $2(66.7 \%)$ \\
\hline Mutant (E-/-) & $1(20.0 \%)$ & $4(80,0 \%)$ \\
\hline \multicolumn{3}{|l|}{ Alleles } \\
\hline Allele E+ & $53(94.6 \%)$ & $40(80.0 \%)$ \\
\hline Allele E- & $3(5.4 \%)$ & $10(20.0 \%)$ \\
\hline HWE (X2/p-value) & $11.75 / 0.0006$ & $14.06 / 0.0002$ \\
\hline
\end{tabular}

BMI: Body mass index, HWE: Hardy Weinberg Equilibrium. 
Table 3. Genotypes and alleles distribution according to demographic cardio-vascular risk factors and according to HIV infection status.

\begin{tabular}{|c|c|c|c|c|}
\hline \multirow{2}{*}{ Age Group (years) $(\mathrm{N}=54)$} & \multicolumn{2}{|l|}{ HIV-Subject } & \multicolumn{2}{|c|}{ ART naive PLHIV } \\
\hline & & & & \\
\hline & $\leq 40(n=18)$ & $>40(\mathrm{n}=13)$ & $\leq 40(\mathrm{n}=11)$ & $>40(\mathrm{n}=12)$ \\
\hline \multicolumn{5}{|l|}{ Genotypes } \\
\hline Wild $(E+/+)$ & $16(57.1 \%)$ & $12(42.9 \%)$ & $8(47.1 \%)$ & $9(52.9 \%)$ \\
\hline Mutant / Wild (E+/-) & $1(50.0 \%)$ & $1(50.0 \%)$ & $0(0.0 \%)$ & $1(100.0 \%)$ \\
\hline Mutant (E-/-) & $1(100.0 \%)$ & $0(0.0 \%)$ & $3(60.0 \%)$ & $2(40.0 \%)$ \\
\hline \multicolumn{5}{|l|}{ Alleles } \\
\hline Allele E+ & $33(91.7 \%)$ & $25(96.2 \%)$ & $16(72.7 \%)$ & $19(79.2 \%)$ \\
\hline Allele E- & $3(8.3 \%)$ & $1(3.8 \%)$ & $6(27.3 \%)$ & $5(20.8 \%)$ \\
\hline HWE (X²/p-value) & $7.290 / 0.0069$ & $0.021 / 0.8853$ & $11.000 / 0.0009$ & $6.700 / 0.0096$ \\
\hline \multicolumn{5}{|l|}{$\operatorname{SEX}(\mathrm{N}=55)$} \\
\hline \multicolumn{5}{|l|}{ Genotypes } \\
\hline Wild $(E+/+)$ & $24(82.8 \%)$ & $5(17.2 \%)$ & $9(52.9 \%)$ & $8(47.1 \%)$ \\
\hline Mutant / Wild (E+/-) & $1(50.0 \%)$ & $1(50.0 \%)$ & $1(100.0 \%)$ & $0(0.0 \%)$ \\
\hline Mutant (E-/-) & $1(100.0 \%)$ & $0(0.0 \%)$ & $3(60.0 \%)$ & $2(40.0 \%)$ \\
\hline \multicolumn{5}{|l|}{ Alleles } \\
\hline Allele E+ & $49(94.2 \%)$ & $11(91.7 \%)$ & $19(73.1 \%)$ & $16(80.0 \%)$ \\
\hline Allele E- & $3(5.8 \%)$ & $1(8.3 \%)$ & $7(26.9 \%)$ & $4(20.0 \%)$ \\
\hline HWE ( $\mathrm{X}^{2} / \mathrm{p}$-value $)$ & $10.860 / 0.0010$ & $0.050 / 0.8238$ & $8.410 / 0.0037$ & $10.000 / 0.0016$ \\
\hline \multicolumn{5}{|l|}{$\operatorname{BMI}\left(\mathrm{kg} / \mathrm{m}^{2}\right)(\mathrm{N}=53)$} \\
\hline & $\leq 25(\mathrm{n}=20)$ & $>25(\mathrm{n}=11)$ & $\leq 25(\mathrm{n}=8)$ & $>25(\mathrm{n}=14)$ \\
\hline \multicolumn{5}{|l|}{ Genotypes } \\
\hline Wild $(\mathrm{E}+/+)$ & $19(67.9 \%)$ & $9(32.1 \%)$ & $7(4102 \%)$ & $10(58.8 \%)$ \\
\hline Mutant / Wild (E+/-) & $1(50.0 \%)$ & $1(50.0 \%)$ & $0(0.0 \%)$ & $1(100.0 \%)$ \\
\hline Mutant (E-/-) & $0(0.0 \%)$ & $1(100.0 \%)$ & $1(25.0 \%)$ & $3(75.0 \%)$ \\
\hline \multicolumn{5}{|l|}{ Alleles } \\
\hline Allele E+ & $39(97.5 \%)$ & $19(86.4 \%)$ & $14(87.5 \%)$ & $21(75.0 \%)$ \\
\hline Allele E- & $1(2.5 \%)$ & $3(13.6 \%)$ & $2(12.5 \%)$ & $7(25.0 \%)$ \\
\hline HWE (X²/p-value) & $0.013 / 0.9087$ & $4.147 / 0,0417$ & $8 / 0.0047$ & $9.175 / 0.0025$ \\
\hline
\end{tabular}

BMI: Body mass index, HWE: Hardy Weinberg Equilibrium.

\subsection{Presentation of the Population According to Serum Lipid and Apoprotein Levels}

Regardless of HIV status, only the genotypic frequencies in subjects with high LDL-C were at HWE (Table 4). The mutant allele was more frequent in subjects with increased TG, LDL-C, Lp (a), atherogenicity index TC/HDL-C (Table 4) and Apo B100 (Table 5) and in those with normal total cholesterolemia, decreased HDL-C (Table 4) and Apo A1 (Table 5). Taking HIV status into account, the mutant allele was more frequent in PLHIV (14\% to $32 \%$ ) than in HIV- subjects ( $2 \%$ to $23 \%$ ) (Table 6, Table $7)$. The mutant allele was more frequent especially in PLHIV with total hypercholesterolemia $(28.1 \%)$, normal LDL cholesterolemia (26.7\%), HDL hypocholesterolemia (27.3\%), hyperapoproteinemia Lp (a) (28.1\%), high atherogenicity index TC/HDL-C (23.7\%) (Table 6), hypoapoproteinemia A1 (32.1\%), hyperapoproteinemia B100 (28.1\%) and high atherogenicity index ApoB/Apo A1 (23.7\%) (Table 7).

Table 4. Genotypes and alleles distribution according to lipid levels in the general population regardless of hiv infection status.

\begin{tabular}{|c|c|c|c|}
\hline PARAMETERS & \multicolumn{3}{|l|}{ CONCENTRATION LEVEL } \\
\hline \multirow[t]{2}{*}{$\mathrm{TG}(\mathrm{g} / \mathrm{L})(\mathrm{N}=55)$} & & & \\
\hline & Low $(<0.3)(\mathrm{n}=1)$ & Normal $(0.3-1.2)(n=19)$ & $\operatorname{High}(>1.2)(\mathrm{n}=35)$ \\
\hline \multicolumn{4}{|l|}{ Genotypes } \\
\hline Wild (E+/+) & $1(2.1 \%)$ & $17(37.0 \%)$ & $28(60.9 \%)$ \\
\hline Mutant / Wild (E+/-) & $0(0.0 \%)$ & $1(33.3 \%)$ & $2(66.7 \%)$ \\
\hline Mutant (E-/-) & $0(0.0 \%)$ & $1(33.3 \%)$ & $5(83.3 \%)$ \\
\hline \multicolumn{4}{|l|}{ Alleles } \\
\hline Allele E+ & $1(100.0 \%)$ & $35(92.1 \%)$ & $58(82.9 \%)$ \\
\hline Allele E- & $0(0.0 \%)$ & $3(7.9 \%)$ & $12(17.1 \%)$ \\
\hline HWE ( $X^{2} / p$-value $)$ & NA & $7.74 / 0.0054$ & $22.35 /<0.0001$ \\
\hline \multicolumn{4}{|l|}{$\mathrm{TC}(\mathrm{g} / \mathrm{L})(\mathrm{N}=55)$} \\
\hline \multicolumn{4}{|l|}{ Genotypes } \\
\hline Wild $(\mathrm{E}+/+)$ & $1(2.2 \%)$ & $30(65.2 \%)$ & $15(32.6 \%)$ \\
\hline Mutant / Wild (E+/-) & $0(0.0 \%)$ & $2(66.7 \%)$ & $1(33.3 \%)$ \\
\hline Mutant (E-/-) & $0(0.0 \%)$ & $5(83.3 \%)$ & $1(16.7 \%)$ \\
\hline
\end{tabular}




\begin{tabular}{|c|c|c|c|}
\hline PARAMETERS & CONCENTRATION LEVEL & & \\
\hline \multicolumn{4}{|l|}{ Alleles } \\
\hline Allele E+ & $2(100.0 \%)$ & $62(83.8 \%)$ & $31(91.2 \%)$ \\
\hline Allele E- & $0(0.0 \%)$ & $12(16.2 \%)$ & $3(8.8 \%)$ \\
\hline HWE (X²/p-value) & NA & $23.74 /<0.0001$ & $6.84 / 0.0089$ \\
\hline \multicolumn{4}{|l|}{ LDL-C $(\mathrm{g} / \mathrm{L})(\mathrm{N}=55)$} \\
\hline \multicolumn{4}{|l|}{ Genotypes } \\
\hline Wild $(E+/+)$ & $35(76.1 \%)$ & & $11(23.9 \%)$ \\
\hline Mutant / Wild (E+/-) & $1(33.3 \%)$ & & $2(66.7 \%)$ \\
\hline Mutant (E-/-) & $5(83.3 \%)$ & & $1(16.7 \%)$ \\
\hline \multicolumn{4}{|l|}{ Alleles } \\
\hline Allele E+ & $71(86.6 \%)$ & & $24(85.7 \%)$ \\
\hline Allele E- & $11(13.4 \%)$ & & $4(14.3 \%)$ \\
\hline HWE ( $\mathrm{X}^{2} / \mathrm{p}$-value $)$ & $32.84 /<0.0001$ & & $2.43 / 0.119$ \\
\hline \multicolumn{4}{|l|}{ HDL-C $(g / L)(N=55)$} \\
\hline & Low $(<0.40)(\mathrm{n}=20)$ & & Normal $(\geq 0.40)(n=35)$ \\
\hline \multicolumn{4}{|l|}{ Genotypes } \\
\hline Wild $(\mathrm{E}+/+)$ & $15(32.6 \%)$ & & $31(67.4 \%)$ \\
\hline Mutant / Wild (E+/-) & $1(33.3 \%)$ & & $2(66.7 \%)$ \\
\hline Mutant (E-/-) & $4(66.7 \%)$ & & $2(33.3 \%)$ \\
\hline \multicolumn{4}{|l|}{ Alleles } \\
\hline Allele E+ & $31(77.5 \%)$ & & $64(91.4 \%)$ \\
\hline Allele E- & $9(22.5 \%)$ & & $6(8.6 \%)$ \\
\hline HWE (X²/p-value) & $14.68 / 0.0001$ & & $14.13 / 0.0002$ \\
\hline \multicolumn{4}{|c|}{ Lipoprotéen Lp (a) (g/L) (N=55) } \\
\hline & $\operatorname{Normal}(<0.25)(\mathrm{n}=19)$ & & High $(\geq 0.25)(n=36)$ \\
\hline \multicolumn{4}{|l|}{ Genotypes } \\
\hline Wild $(\mathrm{E}+/+)$ & $16(34.8 \%)$ & & $30(65.2 \%)$ \\
\hline Mutant / Wild (E+/-) & $1(33.3 \%)$ & & $2(66.7 \%)$ \\
\hline Mutant (E-/-) & $2(33.3 \%)$ & & $4(66.7 \%)$ \\
\hline \multicolumn{4}{|l|}{ Alleles } \\
\hline Allele E+ & $33(86.8 \%)$ & & $62(86.1 \%)$ \\
\hline Allele E- & $5(13.2 \%)$ & & $10(13.9 \%)$ \\
\hline HWE ( $X^{2} / p$-value $)$ & $11.26 / 0.0008$ & & $21.22 /<0.0001$ \\
\hline \multicolumn{4}{|c|}{ Atherogenicity index $1:$ TC/HDL-C $(\mathrm{N}=55)$} \\
\hline & $\begin{array}{l}\text { Normal }(<3.3[\text { Women] or }<4.4[\text { men] }) \\
(\mathrm{n}=19)\end{array}$ & & $\begin{array}{l}\text { High }(\geq 3.3 \text { [women] or } \geq 4.4 \text { [men]) } \\
(n=36)\end{array}$ \\
\hline \multicolumn{4}{|l|}{ Genotypes } \\
\hline Wild $(\mathrm{E}+/+)$ & $17(37.0 \%)$ & & $29(63.0 \%)$ \\
\hline Mutant / Wild (E+/-) & $1(33.3 \%)$ & & $2(66.7 \%)$ \\
\hline Mutant (E-/-) & $1(16.7 \%)$ & & $5(83.3 \%)$ \\
\hline \multicolumn{4}{|l|}{ Alleles } \\
\hline Allele E+ & $35(92.1 \%)$ & & $60(83.3 \%)$ \\
\hline Allele E- & $3(7.9 \%)$ & & $12(16.7 \%)$ \\
\hline HWE ( $X^{2} / p$-value $)$ & $7.74 / 0.0054$ & & $23.04 /<0.0001$ \\
\hline
\end{tabular}

TG: Triglyceride, TC: Total cholesterol, LDL-C: low density lipoprotein-Cholesterol, HDL-C: high density lipoprotein-Cholesterol, HWE: Hardy Weinberg Equilibrium.

Table 5. Genotypes and alleles distribution according to apoprotein levels in the general population regardless of HIV infection status.

\begin{tabular}{|c|c|c|c|}
\hline PARAMETERS & CONCENTRATION LEVEL & & \\
\hline \multicolumn{4}{|l|}{ Apo A1 (g/L) $(\mathrm{N}=55)$} \\
\hline & Low $(<1.18)(\mathrm{n}=21)$ & $\operatorname{Normal}(1.18-1.46)(\mathrm{n}=19)$ & $\operatorname{High}(>1.46)(n=15)$ \\
\hline \multicolumn{4}{|l|}{ Genotypes } \\
\hline Wild (E+/+) & $14(30.4 \%)$ & $19(41.3 \%)$ & $13(28.3 \%)$ \\
\hline Mutant / Wild (E+/-) & $2(66.7 \%)$ & $0(0.0 \%)$ & $1(33.3 \%)$ \\
\hline Mutant (E-/-) & $5(83.3 \%)$ & $0(0.0 \%)$ & $1(16.7 \%)$ \\
\hline \multicolumn{4}{|l|}{ Alleles } \\
\hline Allele E+ & $30(71.4 \%)$ & $38(100.0 \%)$ & $27(90.0 \%)$ \\
\hline Allele E- & $12(28.6 \%)$ & $0(0.0 \%)$ & $3(10.0 \%)$ \\
\hline HWE ( $X^{2} / p$-value $)$ & $12.34 / 0.0004$ & NA & $5.95 / 0.0147$ \\
\hline \multicolumn{4}{|l|}{ Apo B (g/L) $(\mathrm{N}=55)$} \\
\hline & Low $(<0.50)(\mathrm{n}=2)$ & Normal $(0.50-0.82)(n=9)$ & High $(>0.82)(n=45)$ \\
\hline \multicolumn{4}{|l|}{ Genotypes } \\
\hline Wild (E+/+) & $2(4.3 \%)$ & $7(15.2 \%)$ & $37(80.5 \%)$ \\
\hline Mutant / Wild (E+/-) & $0(0.0 \%)$ & $1(33.3 \%)$ & $3(66.7 \%)$ \\
\hline Mutant (E-/-) & $0(0.0 \%)$ & $1(16.7 \%)$ & $5(83.3 \%)$ \\
\hline
\end{tabular}




\begin{tabular}{|c|c|c|c|}
\hline PARAMETERS & CONCENTRATION LEVEL & & \\
\hline \multicolumn{4}{|l|}{ Alleles } \\
\hline Allele E+ & $4(100 \%)$ & $15(83,3 \%)$ & $77(85.6 \%)$ \\
\hline Allele E- & $0(0 \%)$ & $3(16,7 \%)$ & $13(14.4 \%)$ \\
\hline HWE ( $\mathrm{X}^{2} / \mathrm{p}$-value $)$ & NA & $3,24 / 0,0719$ & $24 /<0.0001$ \\
\hline \multicolumn{4}{|c|}{ Atherogenicity index $2: \mathrm{ApoB} / \mathrm{ApoA} 1(\mathrm{~N}=55)$} \\
\hline & Normal $(0.37-0.63)(n=11)$ & & High $(>0.63)(n=44)$ \\
\hline \multicolumn{4}{|l|}{ Genotypes } \\
\hline Wild $(\mathrm{E}+/+)$ & $9(19.6 \%)$ & & $37(80.4 \%)$ \\
\hline Mutant / Wild (E+/-) & $1(33.3 \%)$ & & $2(66.7 \%)$ \\
\hline Mutant (E-/-) & $1(16.7 \%)$ & & $5(83.3 \%)$ \\
\hline \multicolumn{4}{|l|}{ Alleles } \\
\hline Allele E+ & $19(86.4 \%)$ & & $76(86.4 \%)$ \\
\hline Allele E- & $3(13.6 \%)$ & & $12(13.6 \%)$ \\
\hline HWE ( $\mathrm{X}^{2} / \mathrm{p}$-value $)$ & $4,15 / 0.0417$ & & $28.66 /<0.0001$ \\
\hline
\end{tabular}

HWE: Hardy Weinberg Equilibrium.

Table 6. Genotypes and alleles distribution according to lipid levels and according to HIV infection status.

\begin{tabular}{|c|c|c|c|c|c|c|c|}
\hline \multirow{3}{*}{$\begin{array}{l}\text { PARAMETER } \\
\text { TG }(\mathrm{g} / \mathrm{L})(\mathrm{N}=55)\end{array}$} & \multicolumn{3}{|l|}{ HIV- Subject } & \multicolumn{4}{|c|}{ ART naive PLHIV } \\
\hline & \multirow[b]{2}{*}{$\begin{array}{l}\text { Low }(<0.3) \\
(\mathrm{n}=1)\end{array}$} & \multirow[b]{2}{*}{$\begin{array}{l}\text { Normal }(0.3-1.2) \\
(\mathrm{n}=12)\end{array}$} & \multirow[b]{2}{*}{$\begin{array}{l}\operatorname{High}(>1.2) \\
(\mathrm{n}=19)\end{array}$} & \multirow[b]{2}{*}{$\begin{array}{l}\text { Low }(<0.3) \\
(\mathrm{n}=0)\end{array}$} & \multirow{2}{*}{\multicolumn{2}{|c|}{$\begin{array}{l}\text { Normal }(0.3-1.2) \\
(\mathrm{n}=7)\end{array}$}} & \multirow[b]{2}{*}{$\begin{array}{l}\operatorname{High}(>1.2) \\
(\mathrm{n}=16)\end{array}$} \\
\hline & & & & & & & \\
\hline \multicolumn{8}{|l|}{ Genotypes } \\
\hline Wild $(E+/+)$ & $1(3.5 \%)$ & $11(37.9 \%)$ & $17(58.6 \%)$ & $0(0.0 \%)$ & \multicolumn{2}{|c|}{$6(35.3 \%)$} & $11(64.7 \%)$ \\
\hline Mutant / Wild (E+/-) & $0(0.0 \%)$ & $1(50.0 \%)$ & $1(50.0 \%)$ & $0(0.0 \%)$ & \multicolumn{2}{|c|}{$0(0.0 \%)$} & $1(100.0 \%)$ \\
\hline Mutant (E-/-) & $0(0.0 \%)$ & $0(0.0 \%)$ & $1(100.0 \%)$ & $0(0.0 \%)$ & \multicolumn{2}{|c|}{$1(20.0 \%)$} & $4(80.0 \%)$ \\
\hline \multicolumn{8}{|l|}{ Alleles } \\
\hline Allele E+ & $2(100.0 \%)$ & $23(95.8 \%)$ & $35(92.1 \%)$ & $0(0.0 \%)$ & \multicolumn{2}{|c|}{$12(85.7 \%)$} & $23(71.9 \%)$ \\
\hline Allele E- & $0(0.0 \%)$ & $1(4.2 \%)$ & $3(7.9 \%)$ & $0(0.0 \%)$ & \multicolumn{2}{|c|}{$2(14.3 \%)$} & $9(28.1 \%)$ \\
\hline HWE ( $\mathrm{X}^{2} / \mathrm{p}$-value $)$ & NA & $0.023 / 0.8803$ & $7.74 / 0.0054$ & NA & \multicolumn{2}{|c|}{$7 / 0.0082$} & $11.43 / 0.0007$ \\
\hline $\mathrm{TC}(\mathrm{g} / \mathrm{L})(\mathrm{N}=55)$ & & & & & & & \\
\hline & $\begin{array}{l}\text { Low }(<1.06) \\
(\mathrm{n}=1)\end{array}$ & $\begin{array}{l}\text { Normal }(1.06-2.5) \\
(\mathrm{n}=19)\end{array}$ & $\begin{array}{l}\operatorname{High}(>2.5) \\
(\mathrm{n}=12)\end{array}$ & $\begin{array}{l}\text { Low }(<1.06) \\
(\mathrm{n}=0)\end{array}$ & $\begin{array}{l}\text { Norm } \\
(\mathrm{n}=18\end{array}$ & $-2.5)$ & $\begin{array}{l}\operatorname{High}(>2.5) \\
(\mathrm{n}=5)\end{array}$ \\
\hline Genotypes & & & & & & & \\
\hline Wild (E+/+) & $1(3.5 \%)$ & $17(58.6 \%)$ & $11(37.9 \%)$ & $0(0.0 \%)$ & $13(7$ & & $4(23.5 \%)$ \\
\hline Mutant / Wild (E+/-) & $0(0.0 \%)$ & $1(50.0 \%)$ & $1(50.0 \%)$ & $0(0.0 \%)$ & $1(10$ & & $0(0.0 \%)$ \\
\hline Mutant (E-/-) & $0(0.0 \%)$ & $1(100.0 \%)$ & $0(0.0 \%)$ & $0(0.0 \%)$ & $4(80$ & & $1(20.0 \%)$ \\
\hline Alleles & & & & & & & \\
\hline Allele E+ & $2(100.0 \%)$ & $35(92.1 \%)$ & $23(95.8 \%)$ & $0(0.0 \%)$ & $27(7$ & & $8(80.0 \%)$ \\
\hline Allele E- & $0(0.0 \%)$ & $3(7.9 \%)$ & $1(4.2 \%)$ & $0(0.0 \%)$ & $9(25$ & & $2(20.0 \%)$ \\
\hline HWE ( $\mathrm{X}^{2} / \mathrm{p}$-value $)$ & NA & $7.74 / 0.0054$ & $0.023 / 0.8803$ & NA & 13.06 & & $5 / 0.0253$ \\
\hline LDL-C $(g / L)(N=53)$ & Normal $(<1.6$ & $=26)$ & 60) $(n=6)$ & Normal $(<1.60$ & $1=15)$ & High & 1.60) $(\mathrm{n}=8)$ \\
\hline Genotypes & & & & & & & \\
\hline Wild $(\mathrm{E}+/+)$ & $24(82.8 \%)$ & & & $11(64.7 \%)$ & & $6(35$ & \\
\hline Mutant / Wild (E+/-) & $1(50.0 \%)$ & & & $0(0.0 \%)$ & & $1(10$ & $\%)$ \\
\hline Mutant (E-/-) & $1(100.0 \%)$ & & & $4(80.0 \%)$ & & $1(20$ & \\
\hline Alleles & & & & & & & \\
\hline Allele E+ & 49 (94.2\%) & & & $22(73.3 \%)$ & & $13(8$ & $\%)$ \\
\hline Allele E- & $3(5.8 \%)$ & & & $8(26.7 \%)$ & & $3(18$ & \\
\hline HWE ( $\mathrm{X}^{2} / \mathrm{p}$-value $)$ & $10.86 / 0.0010$ & & 8238 & $15 / 0.0001$ & & 2.78 & 095 \\
\hline HDL-C $(\mathrm{g} / \mathrm{L})(\mathrm{N}=55)$ & Low $(<0.40)($ & & $>0.40)(n=23)$ & Low $(<0.40)($ & & Norm & $(\geq 0.40)(\mathrm{n}=12)$ \\
\hline Genotypes & & & & & & & \\
\hline Wild $(E+/+)$ & $7(24.1 \%)$ & & & $8(47.1 \%)$ & & $9(52$ & \\
\hline Mutant / Wild (E+/-) & $1(50.0 \%)$ & & & $0(0.0 \%)$ & & $1(10$ & $\%)$ \\
\hline Mutant (E-/-) & $1(100.0 \%)$ & & & $3(60.0 \%)$ & & $2(40$ & \\
\hline Alleles & & & & & & & \\
\hline Allele E+ & $15(83.3 \%)$ & & & $16(72.7 \%)$ & & $19(7$ & $\%)$ \\
\hline Allele E- & $3(16.7 \%)$ & & & $6(27.3 \%)$ & & $5(20$ & \\
\hline HWE ( $\mathrm{X}^{2} / \mathrm{p}$-value $)$ & $3.24 / 0.0719$ & & 9151 & $11 / 0.0009$ & & 6.70 & 0096 \\
\hline Lipoprotéen Lp (a) (g & $(\mathrm{N}=55)$ & & & & & & \\
\hline & Normal $(<0.2$ & $=12)$ & 25) $(n=20)$ & Normal $(<0.25$ & 1=7) & High & $0.25)(\mathrm{n}=16)$ \\
\hline Génotypes & & & & & & & \\
\hline Wild $(E+/+)$ & $10(34.5 \%)$ & & & $6(35.3 \%)$ & & $11(6$ & $\%)$ \\
\hline Mutant / Wild (E+/-) & $1(50.0 \%)$ & & & $0(0.0 \%)$ & & $1(10$ & $\%)$ \\
\hline Mutant (E-/-) & $1(100.0 \%)$ & & & $1(20.0 \%)$ & & $4(80$ & \\
\hline
\end{tabular}




\begin{tabular}{|c|c|c|c|c|}
\hline \multirow{2}{*}{$\begin{array}{l}\text { PARAMETER } \\
\text { Alleles }\end{array}$} & \multicolumn{2}{|l|}{ HIV-Subject } & \multicolumn{2}{|l|}{ ART naive PLHIV } \\
\hline & & & & \\
\hline Allele E+ & $21(87.5 \%)$ & $39(97.5 \%)$ & $12(85.7 \%)$ & $23(71.9 \%)$ \\
\hline Allele E- & $3(12.5 \%)$ & $1(2.5 \%)$ & $2(14.3 \%)$ & $9(28.1 \%)$ \\
\hline HWE ( $\mathrm{X}^{2} / \mathrm{p}$-value $)$ & $4.60 / 0.0320$ & $0,013 / 0,9087$ & $7 / 0.0082$ & $11.44 / 0.0007$ \\
\hline \multicolumn{5}{|c|}{ Atherogenivity index 1 : TC/HDL-C $(\mathrm{N}=55)$} \\
\hline & $\begin{array}{l}\text { Normal }(<3.3 \text { [women] or }<4.4 \\
[\mathrm{men}])(\mathrm{n}=15)\end{array}$ & $\begin{array}{l}\text { High }(\geq 3.3 \text { [women] or } \geq 4.4 \\
{[\text { homme] })(n=17)}\end{array}$ & $\begin{array}{l}\text { Normal }(<3.3 \text { [women] or }<4.4 \\
\text { men] })(n=4)\end{array}$ & $\begin{array}{l}\operatorname{High}(\geq 3.3 \text { [women] or } \\
\geq 4.4[\text { men] })(n=19)\end{array}$ \\
\hline \multicolumn{5}{|l|}{ Genotypes } \\
\hline Wild $(E+/+)$ & $14(48.3 \%)$ & $15(51.7 \%)$ & $3(17.6 \%)$ & $14(82.4 \%)$ \\
\hline Mutant / Wild (E+/-) & $1(50.0 \%)$ & $1(50.0 \%)$ & $0(0.0 \%)$ & $1(100.0 \%)$ \\
\hline Mutant (E-/-) & $0(0.0 \%)$ & $1(100.0 \%)$ & $1(20.0 \%)$ & $4(80.0 \%)$ \\
\hline \multicolumn{5}{|l|}{ Alleles } \\
\hline Allele E+ & $29(96.7 \%)$ & $31(91.2 \%)$ & $6(75.0 \%)$ & $29(76.3 \%)$ \\
\hline Allele E- & $1(3.3 \%)$ & $3(8.8 \%)$ & $2(25.0 \%)$ & $9(23.7 \%)$ \\
\hline HWE ( $X^{2} / p$-value $)$ & $0.018 / 0.8938$ & $6.84 / 0.0089$ & $4 / 0.0455$ & $13.87 / 0.0002$ \\
\hline
\end{tabular}

TG: Triglyceride, TC: Total cholesterol, LDL-C: low density lipoprotein-Cholesterol, HDL-C: high density lipoprotein-Cholesterol, HWE: Hardy Weinberg Equilibrium.

Table 7. Genotypes and alleles distribution according to apoprotein levels and according to HIV infection status.

\begin{tabular}{|c|c|c|c|c|c|c|c|}
\hline \multirow{2}{*}{$\begin{array}{l}\text { PARAMETER } \\
\text { Apo } \mathrm{A} 1(\mathrm{~g} / \mathrm{L})(\mathrm{N}=55)\end{array}$} & \multicolumn{4}{|l|}{ HIV- Subject } & \multicolumn{3}{|c|}{ ART naive PLHIV } \\
\hline & \multirow[b]{2}{*}{$\begin{array}{l}\text { Low }(<1.18) \\
(\mathrm{n}=7)\end{array}$} & & & & \multirow[b]{2}{*}{$\begin{array}{l}\text { Low }(<1.18) \\
(\mathrm{n}=14)\end{array}$} & & \\
\hline & & \multicolumn{2}{|c|}{$\begin{array}{l}\text { Normal }(1.18-1.46) \\
(\mathrm{n}=11)\end{array}$} & $\begin{array}{l}\operatorname{high}(>1.46) \\
(\mathrm{n}=14)\end{array}$ & & $\begin{array}{l}\text { Normal }(1.18-1.46) \\
(\mathrm{n}=8)\end{array}$ & $\begin{array}{l}\text { High }>1.46) \\
(n=1)\end{array}$ \\
\hline \multicolumn{8}{|l|}{ Génotypes } \\
\hline Genotypes & $5(17.2 \%)$ & \multicolumn{2}{|l|}{$11(37.9 \%)$} & $13(44.8 \%)$ & $9(52.9 \%)$ & $8(47.1 \%)$ & $0(0.0 \%)$ \\
\hline Wild $(E+/+)$ & $1(50.0 \%)$ & \multicolumn{2}{|l|}{$0(0.0 \%)$} & $1(50.0 \%)$ & $1(100.0 \%)$ & $0(0.0 \%)$ & $0(0.0 \%)$ \\
\hline Mutant / Wild (E+/-) & $1(100.0 \%)$ & \multicolumn{2}{|l|}{$0(0 \%)$} & $0(0.0 \%)$ & $4(80.0 \%)$ & $0(0.0 \%)$ & $1(20.0 \%)$ \\
\hline \multicolumn{8}{|l|}{ Mutant (E-/-) } \\
\hline Alleles & $11(78.6 \%)$ & \multicolumn{2}{|l|}{$22(100.0 \%)$} & 27 (96.4\%) & $19(67.9 \%)$ & $16(100.0 \%)$ & $0(0.0 \%)$ \\
\hline Allele E+ & $3(21.4 \%)$ & \multicolumn{2}{|l|}{$0(0.0 \%)$} & $1(3.6 \%)$ & $9(32.1 \%)$ & $0(0 / 0 \%)$ & $2(100.0 \%)$ \\
\hline \multirow{3}{*}{$\begin{array}{l}\text { Allele E- } \\
\text { Apo B (g/L) }(\mathrm{N}=55)\end{array}$} & $2.32 / 0.1277$ & \multirow{2}{*}{\multicolumn{2}{|c|}{ NA }} & $0.019 / 0.8898$ & $9.79 / 0.0018$ & \multirow[t]{2}{*}{ NA } & NA \\
\hline & & & & & & & \\
\hline & $\begin{array}{l}\text { Low }(<0.50) \\
(n=1)\end{array}$ & \multicolumn{2}{|c|}{$\begin{array}{l}\text { Normal }(0.50-0.82) \\
(n=3)\end{array}$} & $\begin{array}{l}\operatorname{High}(>0.82) \\
(\mathrm{n}=28)\end{array}$ & $\begin{array}{l}\text { Low }(<0.50) \\
(\mathrm{n}=1)\end{array}$ & $\begin{array}{l}\text { Normal }(0.50-0.82) \\
(n=6)\end{array}$ & $\begin{array}{l}\operatorname{High}(>0.82) \\
(\mathrm{n}=16)\end{array}$ \\
\hline \multicolumn{8}{|l|}{ Genotypes } \\
\hline Wild $(E+/+)$ & $1(3.4 \%)$ & \multicolumn{2}{|l|}{$2(6.9 \%)$} & $26(89.7 \%)$ & $1(5.9 \%)$ & $5(29.4 \%)$ & $11(64.7 \%)$ \\
\hline Mutant / Wild (E+/-) & $0(0.0 \%)$ & \multicolumn{2}{|l|}{$1(50.0 \%)$} & $1(50.0 \%)$ & $0(0.0 \%)$ & $0(0.0 \%)$ & $1(100.0 \%)$ \\
\hline Mutant (E-/-) & $0(0.0 \%)$ & $0(0.0 \%)$ & & $1(100.0 \%)$ & $0(0.0 \%)$ & $1(20.0 \%)$ & $4(80.0 \%)$ \\
\hline Alleles & & & & & & & \\
\hline Allele E+ & $2(100.0 \%)$ & $5(83.3 \%)$ & & $53(94.6 \%)$ & $2(100.0 \%)$ & $10(83.3 \%)$ & $23(71.9 \%)$ \\
\hline Allele E- & $0(0.0 \%)$ & $1(16.7 \%)$ & & $3(5.4 \%)$ & $0(0.0 \%)$ & $2(16.7 \%)$ & $9(28.1 \%)$ \\
\hline HWE ( $X^{2} / p$-value $)$ & NA & $0.12 / 0.7290$ & & $11.75 / 0.0006$ & NA & $6 / 0.0143$ & $11.43 / 0.0007$ \\
\hline Atherogenivity index & ApoB/ApoA1 (I & & & & & & \\
\hline & Normal $(0.37$ & 63) $(n=7)$ & High & $.63)(n=25)$ & Normal $(0.37$ & High $(>$ & $(n=19)$ \\
\hline Genotypes & & & & & & & \\
\hline Wild $(E+/+)$ & $6(20.7 \%)$ & & $23(7$ & & $3(17.6 \%)$ & $14(82.4$ & \\
\hline Mutant / Wild (E+/-) & $1(50.0 \%)$ & & $1(50$ & & $0(0.0 \%)$ & $1(100.0$ & \\
\hline Mutant (E-/-) & $0(0.0 \%)$ & & $1(10$ & & $1(20.0 \%)$ & $4\left(80.0^{\circ}\right.$ & \\
\hline Alleles & & & & & & & \\
\hline Allele E+ & $13(92.9 \%)$ & & $47(9$ & & $6(75.0 \%)$ & $29(76.3$ & \\
\hline Allele E- & $1(7.1 \%)$ & & $3(6.0$ & & $2(25.0 \%)$ & $9\left(23.7^{\circ}\right.$ & \\
\hline HWE ( $\mathrm{X}^{2} / \mathrm{p}$-value $)$ & $0.041 / 0.8387$ & & 10.41 & .0013 & $4 / 0.0455$ & $13.87 /(\mathrm{c} / \mathrm{r} / \mathrm{s}$ & \\
\hline
\end{tabular}

HWE: Hardy Weinberg Equilibrium.

\section{Discussion}

This cross-sectional analytical study evaluated the impact of EcoR1 polymorphism on the occurrence of dyslipidemia during HIV1 infection not treated with antiretroviral drugs. It was carried out on 55 subjects including 23 ART-naive
PLHIV (41.81\%) and 32 HIV negative control subjects (58.18\%).

High BMI was more common in PLHIV (63.6\%) $(p=0.043 \%)$ and so was the average BMI $(p=0.046)$. In contrast, Muhammad et al. [19] as well as Ogunmola et al. [20] reported normal average BMIs $\left(22.0\right.$ and $\left.22.6 \mathrm{~kg} / \mathrm{m}^{2}\right)$ in 
ART-naive PLHIV with lower proportions of overweight/obese subjects of $22 \%$ and $3 \%$ respectively. This difference could be explained by the dietary habits of the study populations or by the presence of predisposing factors in the subjects recruited in our study.

PLHIV showed some same lipid disorders as HIVsubjects, including hypertriglyceridemia, high Apo B and Lp (a) concentrations, and high atherogenicity indexes. However, PLHIV showed low Apo A1 concentrations ( $p<$ $0.0001)$ and HDL-C levels $(p=0.016)$. In their review of the literature, Green et al. [21] reported low serum TC, HDL-C and LDL-C concentrations and hypertriglyceridemia in ART-naive PLHIV. A study performed in Togo found higher proportions of PLHIV with hypercholesterolemia (41.4\%) and lower proportions of subjects with hyper LDL-cholesterolemia $(23.5 \%)$ and hypo HDL-cholesterolemia (17.4\%) [2]. It has been found $64.4 \%$ hypo-HDL-cholesterolemia in untreated PLHIV [3] Other authors found, contrary to us, hyperapoproteinemia A1 [10]. These differences could be explained by the features of the study populations. Indeed, Agbeko et al. explored lipid disorders in PLHIV on ART for at least 12 months [2] while untreated PLHIV constituted the population in our study and that of Appiah et al. [3] evaluated antiretroviral molecules influencing lipid level. The difference in nutritional habits of the study populations could explain these discrepancies because lipid status is correlated with diet. Genetic predisposition factors, which themselves vary according to the geographical location of the subjects, could contribute to this variability. These differences in profiles could also be due to the stages of immunodepression of the patients. Indeed, it has been shown that HIV infection induces an early decrease in cholesterol generally affecting TC first, followed by HDL-C and then LDL-C, and a late increase in $\mathrm{TG}$ and these changes are correlated with the degree of immunosuppression in a number of cases [22].

All 3 possible genotypes of EcoR1 polymorphism were found. In contrast, Kone et al. (2018) in Côte d'Ivoire [12] and Sharma et al. in India [11] did not find the mutant genotype (E-/-) in their studies. This could be attributed to the size of the population but also to the geographical location of the population. The HWE was deviated in both HIV- subjects $\left(\mathrm{X}^{2}=6.97 ; p=0.008\right)$ and PLHIV $\left(\mathrm{X}^{2}=19.20\right.$; $p<0.0001)$. This result is consistent with that of Kodogo et al. [23]. The mutant genotype was more common in PLHIV $(85.7 \%)$. The frequency of the mutant allele (E-) was $27.1 \%$ and significantly higher in PLHIV. Kodogo et al. found only $1.2 \%$ of PLHIV with the mutant genotype and a lower frequency of mutant allele (E-) (15\%) [23].

The results observed in the general population differed from those observed in each group. Thus, the division into PLHIV and HIV- subjects allowed us to clarify the impact of the EcoR1 polymorphism in PLHIV. Mutant and heterozygous genotypes as well as E- allele (20\%) predominated in subjects with high BMI. The presence of Eallele appeared to be associated with the occurrence of obesity/overweight. In a study made in India [24] and an other in Singapore [25] found no association between EcoR1 polymorphism and obesity.

The mutant allele was more frequent especially in PLHIV with total hypercholesterolemia (28.1\%), normal LDL cholesterol (26.7\%), HDL hypocholesterolemia (27.3\%), hypoapoproteinemia A1 (32.1\%), hyperapoproteinemia B100 (28.1\%), hyper Lp (a) (28.1\%), and high atherogenicity indices $(23.7 \%)$. Kone et al. [13] showed in 2017 that mutations in the Apo B100 gene were more common in PLHIV. Furthermore, the association between the EcoR1 polymorphism and serum lipid variations depends on the lipid and the study population. As a matter of fact, regardless of the subject genotype, the majority of subjects, both healthy and PLHIV showed hypertriglyceridemia, normal TC concentration, high Apo B and Lp (a) values, and high atherogenicity index. The EcoR1 polymorphism did not appear to have an impact on these parameters. In their study, Sharma et al. showed hyperApo B only in E+/- subjects [11]. For $\mathrm{Gu} \mathrm{W}$ et al., serum TC was significantly higher in subjects carrying E- allele [26]. For $\mathrm{Hu} \mathrm{P}$ et al., subjects with the homozygous mutant genotype (E-/-) showed high serum Lp (a) and TC concentrations [9].

Serum LDL-cholesterolemia were high in heterozygotes $(\mathrm{E}+/-)$. However, since HWE is respected, the carrying of Eallele would not expose the subject to high LDL-C concentration. This result was in contrast to that found in a Chinese study which showed that the mutant allele was correlated with high LDL concentrations [26]. However, low HDL-C were found in mutants. Hypoapoproteinemia A1 was predominant in mutant and heterozygous genotypes. The carrying of E- allele would promote a decrease in serum Apo A1 responsible for the low HDL-C concentrations in E-/homozygotes; also found by $\mathrm{Gu} \mathrm{W}$ et al. [26]. In contrast, $\mathrm{Hu}$ P's team [9] reported HDL hypercholesterolemia and hyperApoproteinemia A1 in E-/- homozygotes. These results change from one study to another and this discordance may be induced by the variability due to the study population (age, origin, etc.) but also to the sample size. The interaction of these factors with other genetic, environmental or nutritional factors could alter the effect of the polymorphism [11, 12, 27].

\section{Conclusion}

The results observed in the general population differed from those in PLHIV. The distribution of alleles was not random, and mutant allele (E-) was more frequent in PLHIV and exposed them to high BMI, increased atherogenic apolipoproteins, atherogenicity index and decreased protective apolipoproteins. In order to investigate the link between the effect of ART and Apoproteins B100 and A1 genes polymorphisms, we are considering doing analyses on a population of PLHIV on ART.

\section{Acknowledgements}

We are grateful to $\mathrm{CeDReS}$ for providing the work 
platform, to the staff of CNTS (National Center for Bood Transfusion) for access to the study population and their files, and to the individuals who participated in the study.

\section{References}

[1] ONUSIDA. (2021). FACT SHEET 2021 - Latest global and regional statistics on the status of the AIDS. URL: https://www.unaids.org/sites/default/files/media_asset/UNAID S_FactSheet_fr.pdf; [consulted on june $25^{\text {th }} 202 \overline{1}$ ].

[2] Agbeko D. K., Toyi T., Lihanimpo D., Dzidzonu N. K., Laconi, K., Abago B., \& Awalou, D. M. (2019). Troubles lipidiques et glucidiques à risque cardio-vasculaires chez les personnes vivant avec le virus d'immunodéficience humaine sous traitement antirétroviral: cas du centre de prise en charge médicale de l'ONG Espoir-Vie-Togo à Lomé. The Pan African Medical Journal, 2019; 34: 203. doi: 10.11604/pamj.2019.34.203.20600.

[3] Appiah L. T., Sarfo F. S., Huffman, M. D., Nguah S. B., \& Stiles J. K. (2019). Cardiovascular risk factors among Ghanaian patients with HIV : A cross-sectional study. Clinical Cardiology, 42 (12), 1195-1201.

[4] Dave, J. A., Levitt, N. S., Ross, I. L., Lacerda, M., Maartens, G., \& Blom, D. (2016). Anti-Retroviral Therapy Increases the Prevalence of Dyslipidemia in South African HIV-Infected Patients. PloS One, 11 (3), e0151911.

[5] Aw F, Mingou J, Dioum M, Sarr SA, Leye M, Coly S, Ndiaye MB, Bodian M, Ngaido AA, Mbaye A, Kane A, A Kane A, Ly F, Diao M, Ba SA. (2017). Facteurs de risque cardio-vasculaire chez les patients vivant avec le VIH. Revue Africaine et Malgache de Recherche Scientifique/Sciences de la Santé, 5 (2).

[6] Boccara F, Capeau J, Caron M, Vigouroux C, Cohen A. (2009) VIH, antirétroviraux, dyslipidémie et risque cardiovasculaire. Médecine des Maladies Métaboliques; 3 (1): 59-64.

[7] Alves E. S, Henriques A. D., Tonet-Furioso A. C., Paula R. S., Gomes L. O., Moraes C. F., et Nóbrega O. T. (2020). The APOB rs693 polymorphism impacts the lipid profile of Brazilian older adults. Brazilian Journal of Medical and Biological Research, 53 (3).

[8] Cambien F. (1989). Polymorphisme génétique des apolipoprotéines. Médecine/sciences.; 5 (6): 379-88.

[9] Hu P., Qin Y. H., Jing C. X., Lu, L., Hu, B., \& Du, P. F. (2009) Effect of apolipoprotein B polymorphism on body mass index, serum protein and lipid profiles in children of Guangxi. China. Annals of Human Biology, 36 (4), 411-420.

[10] Kallel, A., Jemaa R., Feki M., Asmi M. E., Souissi M., Sanhaji H., Haj-Taieb S., Omar S., \& Kaabachi N. (2007). Polymorphisme XbaI du gène de l'apolipoprotéine $\mathrm{B}$ dans une population tunisienne: Fréquences alléliques et relation avec les paramètres lipidiques plasmatiques. Annales de Biologie Clinique, 65 (3), 265-270.

[11] Sharma, R., Mahajan, M., Singh, B., Singh, G., \& Singh, P. (2011). Role of the APOB Gene Polymorphism (c.12669G $>$ A, p. Gln4154Lys) in Coronary Artery Disease in the Indian Punjabi Population. Balkan Journal of Medical Genetics: BJMG, 14 (2): 35-40.
[12] Koné F, Edjème-Aké A, Toni T, Anné BJ, Hauhouot-Attoungbré M, Ahiboh H, Djaman A. J and Monnet D. (2018). Xba 1 and Ecor 1 polymorphisms of apolipoprotein b100 gene and lipids abnormalities in HIV infected patients. Int J Recent Sciences Research., 9 (9): 28854-8.

[13] Kone F, Toni T, Edjeme-Ake A, Ahiboh V, Djaman J, Monnet D. (2017). Mutations du gène d'apoprotéine B100 au cours des dyslipidémies chez les sujets vivant avec le VIH. Internationel Journal of Biological and Chemical Sciences., 11: 2366 .

[14] Liu F.-L., Lu W.-B., \& Niu, W.-X. (2010). XbaI polymorphisms of apolipoprotein B gene : Another risk factor of gallstone formation after radical gastrectomy. World Journal of Gastroenterology: WJG, 16 (20), 2549-2553.

[15] Biswas S., Ghoshal, P. K., Halder, B., Ganguly, K., DasBiswas, A., \& Mandal, N. (2013). Apolipoproteins AI/B/E gene polymorphism and their plasma levels in patients with coronary artery disease in a tertiary care-center of Eastern India. Indian Heart Journal, 65 (6): 658-665.

[16] [Bacaër, N. (2011). The Hardy-Weinberg law (1908). In N. Bacaër (Éd.), A Short History of Mathematical Population Dynamics, Springer, Londres (Royaume Uni), p. 59-63.

[17] Oliver M. (2012). A Century of Hardy-Weinberg Equilibrium. Twin Research and Human Genetics, 11 (3): 249-256.

[18] Rehman, A.-, Iqbal, J., Shakeel, A., Qamar, Z. ul, \& Rana, P. (2020). Hardy-Weinberg equilibrium study of six morphogenetic characters in a population of Punjab, Pakistan. All Life, 13 (1): 213-222.

[19] Muhammad, F., Uloko, A., Ramalan, M., Adenike, E., Mukhtar, R., \& Muhammad, F. (2015). Prevalence and risk factors for obesity among HIV patients in Kano, north western Nigeria. Endocrine Abstracts, 38, 285p.

[20] Ogunmola, O. J., Oladosu, O. Y., \& Olamoyegun, A. M. (2014). Association of hypertension and obesity with HIV and antiretroviral therapy in a rural tertiary health center in Nigeria : A cross-sectional cohort study. Vascular Health and Risk Management, 10, 129-137.

[21] Green, M. L. (2002). Evaluation and Management of Dyslipidemia in Patients with HIV Infection. Journal of General Internal Medicine, 17 (10), 797-810.

[22] Daniyam, C., \& Iroezindu, M. (2013). Lipid Profile of Anti-Retroviral Treatment-Naïve HIV-Infected Patients in Jos, Nigeria. Annals of Medical and Health Sciences Research, 3 (1): 26-30.

[23] Kodogo, V., Zhou, D. T., Oektedalen, O., Duri, K., Stray-Pedersen, B., \& Gomo, E. (2016). Apolipoprotein B Gene Polymorphisms and Dyslipidemia in HIV Infected Adult Zimbabweans. The Open AIDS Journal, 10: 190-198.

[24] Misra, A., Nishanth, S., Pasha, S. T., Pandey, R. M., Sethi, P., \& Rawat, D. S. (2001). Relationship of Xba1 and EcoR1 polymorphisms of apolipoprotein-B gene to dyslipidemia and obesity in Asian Indians in North India. Indian Heart Journal, 53 (2): 177-183.

[25] Saha, N., Tay, J. S., Heng, C. K., \& Humphries, S. E. (1993). DNA polymorphisms of the apolipoprotein B gene are associated with obesity and serum lipids in healthy Indians in Singapore. Clinical Genetics, 44 (3): 113-120. 
[26] Gu, W., Zhang, M., Wen, S. (2015). Association between the APOB XbaI and EcoRI polymorphisms and lipids in Chinese: a meta-analysis. Lipids

Health

Dis

14.
[27] Gu, Q.-L., Han, Y., Lan, Y.-M., Li, Y., Kou, W., Zhou, Y.-S., Hai, X.-J., Yan, B., Ci, C.-H. (2017). Association between polymorphisms in the APOB gene and hyperlipidemia in the Chinese Yugur population. Braz J Med Biol Res 50, e6613. https://doi.org/10.1590/1414-431X20176613 